\title{
THE GENERAL SOLUTION OF A FIRST ORDER DIFFERENTIAL POLYNOMIAL
}

\author{
RICHARD M. COHN $^{1}$
}

\begin{abstract}
A purely algebraic proof is given of a theorem, proved analytically by Ritt, which determines the number of derivations needed to find a basis for the perfect ideal of the general solution of an algebraically irreducible first order differential polynomial.
\end{abstract}

1. It was shown by J. F. Ritt $[2,129]$ that to separate the singular components from the general solution of an irreducible first order differential polynomial $G$ of degree $m$ in the first derivative of the indeterminate it is sufficient to decompose the system formed by $G$ and its first $m-1$ derivatives treated as algebraic polynomials. His proof uses analytic methods. Kolchin [1, p. xiv] observed that this result is valid in abstract differential algebra because of the "differential Lefschetz principle" due to Seidenberg [3, p. 160, embedding theorem], but that no purely algebraic proof is known. In this note I give such a proof of Ritt's theorem. The mechanics of the argument are the same as in Ritt's work, except that I have preferred to introduce a parameter to permit power series with integral rather than fractional exponents.

2. Let $K$ be an ordinary differential field of characteristic 0 with derivation $D$, and let $Y$ be a differential indeterminate over $K$. Derivatives in $K\{Y\}$ will frequently be denoted by subscripts.

Theorem. Let $G \in K\{Y\}$ be of order 1 and degree $m$ in $Y_{1}$. Let $G$ have no factor of order 0 and no factor in common with $\partial G / \partial Y_{1}$. Let $P_{1}, \ldots, P_{k}$ be those minimal prime divisors of the ideal $\left(G, \ldots, G_{m-1}\right)$ of $K\left[Y, \ldots, Y_{m}\right]$ which do not contain $\partial G / \partial Y_{1}$. Then no solution in a component of order 0 of the manifold of $G$ as a differential polynomial annuls any $P_{i}$.

Remark. If $G$ is irreducible, then $k=1$ and $P_{1}$ is the intersection with $K\left[Y, \ldots, Y_{m}\right]$ of the differential ideal of the general solution of $G$ [2, p. 130]. The components of order 0 are, of course, precisely the singular components. It follows readily that in the general case $k$ is the number of irreducible factors of $G$, and each $P_{i}$ is the intersection with $K\left[Y, \ldots, Y_{m}\right]$ of the differential ideal of the general solution of an irreducible factor. It follows

Received by the editors January 2, 1975

AMS (MOS) subject classifications (1970). Primary $12 \mathrm{H} 05$.

Key words and phrases. General solution, singular components, basis for a perfect differential ideal.

${ }^{1}$ The author is grateful to the Rutgers University Faculty Academic Study Plan for leave which has aided in the preparation of this paper.

(C) American Mathematical Society 1976 
further that no $P_{i}$ contains a polynomial of order 0 .

Proof. Let $a$ be a solution in a zero order component of the manifold of $G$. We shall assume that $a$ annuls some $P_{i}$, say $P_{1}$, and obtain a contradiction. Note that without loss of generality we may assume $a=0$. Indeed, if we enlarge $K$ to a differential field $K_{1}$ the hypotheses concerning factorization of $G$ remain valid in $K_{1}\{Y\}$. From standard results concerning the effect of ground field extensions we see that $P_{1}$ splits into prime components in $K_{1}\left[Y, \ldots, Y_{m}\right]$ no one of which contains $\partial G / \partial Y_{1}$. The analogous theorem of differential algebral [1, Chapter III, Proposition 3] shows that $a$ is in a zero order component of $G$ as a polynomial in $K_{1}\{Y\}$. Choosing $K_{1}$ to contain $a$ and then making the substitution $Y \rightarrow Y+a$ accomplishes the desired reduction. Henceforth, let $a=0$.

We introduce a parameter $t$ and form the power series ring $K[[t]]$ and its quotient field $K((t))$. These are not differential. However, for $f \in K((t))$ define $\partial f$ to be the result of applying the derivation $D$ of $K$ to every coefficient of $f$, and $d f / d t$ to be the formal derivative of $f$ with respect to $t$. Let $h \in K((t))$. Define $D_{h} f=\partial f+h d f / d t$. Then $D_{h}$ is a derivation of $K((t))$.

Since $P_{1}$ contains no polynomial of order $0, Y \partial G / \partial Y_{1} \notin P_{1}$. By hypothesis $P_{1}$ admits the solution $Y_{i}=0,0 \leqslant i \leqslant m$. It follows that $P_{1}$ has a solution $Y_{i}=f_{i}, 0 \leqslant i \leqslant m$, not annulling $Y \partial G / \partial Y_{1}$, where the $f_{i}$ are in $K((t))$ and begin with terms of positive degree. Concerning this solution we make the following observations.

(a) The $f_{i}$ annul $G_{1}, \ldots, G_{m-1}$. This requirement uniquely determines $f_{2}, \ldots, f_{m}$ when $f_{0}$ and $f_{1}$ are given. This is so since for $1 \leqslant i \leqslant m-1$, $G_{i}=Y_{i+1} \partial G / \partial Y_{1}+$ terms free of $Y_{j}, j>i$, and since $f_{0}, f_{1}$ do not annul $\partial G / \partial Y_{1}$.

(b) Choose $h$ so that $D_{h} f_{0}=f_{1}$. (This is possible since $d f_{0} / d t \neq 0$.) Regarding $K((t))$ as a differential field with the derivation $D_{h}$ we see that $f_{0}$ is a solution of $G$ as a differential polynomial. Hence, $Y_{i}=D_{h}^{i} f_{0}, 0 \leqslant i \leqslant m$, is a solution of the $G_{i}, 0 \leqslant i<m$.

Combining (a) and (b) we have

$$
f_{i}=D_{h}^{i} f_{0}, \quad 0 \leqslant i \leqslant m .
$$

Let $d$ denote the degree of the initial term of $h$. We shall show that $d \leqslant 0$. Let $I$ be the prime differential ideal of $K\{y\}$ with generic zero $f_{0}$. (The derivation is, of course, $D_{h}$.) Since $f_{0}$ actually involves $t, I$ is not of order 0 . Since $G \in I$, this shows that $I$ is of order 1 and is the ideal of a component of the manifold of $G$. If $d>0$, then it is easy to see that each $D_{h}^{i} f_{0}, i=0,1$, $2, \ldots$, begins with a term of positive degree in $t$. This implies that 0 is a solution of $I$, contradicting the assumption that 0 lies in a component of order 0 of the manifold of $G$.

REMARK. One could also show $d \leqslant 0$ (that is, that $f_{1}$ begins with a term of lower degree than $f_{0}$ ) from the fact that $G$ must satisfy the low power condition with respect to $Y$. Conversely, noting that the result $d \leqslant 0$ applies to an arbitrary solution of $G$ (as a polynomial in $Y$ and $Y_{1}$ ) in series of positive powers of $t$, and examining the Newton polygon of $G$, one can prove the necessity of the low power condition for differential polynomials of first order. 
From $d \leqslant 0$ it follows easily that if $f \in K((t))$ has an initial term of degree $a>0$, then $D_{h} f$ has an initial term of degree $a+d-1<a$. Let $f_{0}, f_{1}, f_{m}$ begin with terms of degrees $a, b$, and $c$ respectively. Using (A) and the preceding observation we find $a-b=1-d, a-c=m(1-d)$. That is, $c=a-m(a-b)$.

$G$ must contain two terms with distinct power products $Y^{p} Y_{1}^{q}$ and $Y^{r} Y_{1}^{s}$ which yield terms of the same degree after the substitutions $Y=t^{a}, Y_{1}=t^{b}$. Otherwise $f_{0}, f_{1}$ could not annul $G$. Therefore, $p a+q b=r a+s b$. Let, say, $q>s$. $(q=s$ is not possible, since it implies $r=p$.) Putting $q-s=n$ we rewrite the preceding equation as $n b=(r-p) a$. Then $r-p<n$, since $b<a$. Hence, $n b \leqslant(n-1) a$. By hypothesis $q \leqslant m$, and so $n \leqslant m$. Then $m b \leqslant(m-1) a$. Together with the result of the preceding paragraph this implies $c \leqslant 0$. This is a contradiction, and the proof is complete.

COROLlaRy. Let $G$ be an algebraically irreducible differential polynomial of $K\{Y\}$ which is of order 1 and degree $m$ in $Y_{1}$. Let $I$ be the differential ideal of the general component of the manifold of $G$. Let $P$ be the unique minimal prime divisor of $\left(G, \ldots, G_{m-1}\right)$ which does not contain $\partial G / \partial Y_{1}$. Then $I=\{P\}$, and $I$ has a basis as a perfect differential ideal consisting of polynomials of order not exceeding $m$.

Proof. By [2, p. 30], $I \supset\{P\}$; and, of course, $\{P\} \supset\{G\}$. It follows from the Theorem that no minimal prime divisor of $\{G\}$ except $I$ contains $P$. Hence $I=\{P\}$. A basis for $P$ as a polynomial ideal is a basis for $\{P\}$ as a perfect differential ideal.

\section{REFERENCES}

1. E. R. Kolchin, Differential algebra and algebraic groups, Academic Press, New York and London, 1973.

2. J. F. Ritt, Differential algebra, Amer. Math. Soc. Colloq. Publ., vol. 33, Amer. Math. Soc., Providence, R.I., 1950. MR 12, 7.

3. A. Seidenberg, Abstract differential algebra and the analytic case, Proc. Amer. Math. Soc. 9(1958), 159-164. MR 20 \# 178.

4. - Abstract differential algebra and the analytic case. II, Proc. Amer. Math. Soc. 23(1969), 689-691. MR 40 \#1376.

Department of Mathematics, Rutgers University, New Brunswick, New Jersey 08903 